\title{
Mood instability and reward processing: daily remote monitoring as a modern phenotyping tool
}

for bipolar disorder.

Panchal, P. ${ }^{1}$, Scholl, J. ${ }^{1,2}$, Nelissen, N. ${ }^{1}$, Saunders, KEA. ${ }^{1}$, Darby, D. ${ }^{3}$, Rushworth, MFS. ${ }^{2}$, Harrison, PJ. ${ }^{1}$, Nobre, $\mathrm{AC}^{1,2}$, and Harmer, CJ..

${ }^{I}$ Department of Psychiatry, University of Oxford, Warneford Hospital, Oxford, UK; ${ }^{2}$ Department of Experimental Psychology, University of Oxford, Oxford, UK, ${ }^{3}$ Florey Institute of Neuroscience and Mental Health, The University of Melbourne, Victoria, Australia.

\section{Introduction}

Mood instability is a prominent feature of bipolar disorder (BD) and other affective disorders [1]. Recent research has begun to highlight a strong association between mood instability and cognitive processing, particularly within the decision-making and reward-processing domain, suggesting the ability for mood to bias perception of reward and loss, and to induce risk-taking behaviour [2, 3]. However, whilst this has provided an interesting first-line understanding of the phenotypic nature of $\mathrm{BD}$, research to date has failed to explore the complexities of this relationship over a longitudinal basis. Modern technologies, such as remote online platforms, can address this issue by using high frequency and prospective monitoring. This is particularly important given the dynamic nature of mood instability [4] and the expanding need to understand its underlying neural mechanisms in order to develop new and better-suited targets for treatment [1].

\section{Aims}

Given the emergence of new technologies and the ubiquity of internet access, we aimed to assess whether remote monitoring can capture dynamic mood instability in individuals showing dimensions of BD as measured by the Mood Disorder Questionnaire (MDQ) screening tool, and whether such instability is reflected in risk taking behaviour in a longitudinal decision-making and reward-processing task.

\section{Methods}

Remote mood and cognitive monitoring over 10 weeks, as part of the Cognition and Mood Evolution across Time (COMET) study. We analysed data from 37 participants scoring $>7$ on the MDQ (high MDQ group) and 35 matched controls $(\mathrm{MDQ}<5)$. Mood was assessed on a daily basis using the PANAS scale on an iPad. Participants also completed a decision-making and reward-processing gambling task, "Wheel of Fortune", on the iPad, where they were asked to pick the best out of 2 gambling options, with the aim of winning as many bonus points as possible. Repeated measures ANOVAs were used to analyse mood and cognitive task data.

\section{Results}

The high MDQ group showed greater variability in their PANAS positive affect, $F(1)=10.56, p<0.001$, and negative affect, $F(1)=15.01, p<0.001$, scores compared to the controls. They also reported greater average negative affect scores, $F(1)=14.46, p<0.001$, but were no different in their average positive affect scores.

Whilst the high MDQ participants did not show a significant bias towards riskier decisions in the gambling task in these preliminary analyses, all participants appeared to make less risky choices, $F(5.00)=7.42, p<0.001$, and were less variable in their choices, $F(4.81)=2.50, p<0.05$, across time.

\section{Conclusions}

Longitudinal daily remote mood monitoring captures mood instability in individuals showing dimensions of BD. Concurrent monitoring of cognition, particularly within the reward processing domain, suggests a pattern of dynamic decision-making and warrants further sophisticated analyses to better elucidate the cognitive underpinnings of such mood instability. Nevertheless, findings add to the increasing evidence for mood instability as a marker for BD, and suggest the efficacy of remote monitoring as a modern phenotyping technology in prospectively assessing mood instability and associated cognition. This will aid in the search for biomarkers that can be used in determining the effectiveness of current treatments and in the development of novel therapeutic targets. 


\section{References}

[1] Broome M.R., Saunders K.E.A., Harrison P.J., Marwaha S., 2015. Mood instability: significance, definition and measurement. Br J Psychiatry. Royal College of Psychiatrists; 207(4):283-5.

[2] Eldar E., Rutledge R.B., Dolan R.J., Niv Y., 2016. Mood as Representation of Momentum. Trends Cogn Sci. Elsevier Ltd; 20(1):15-24.

[3] Eldar E., Niv Y., 2015. Interaction between emotional state and learning underlies mood instability. Nat Commun. Nature Publishing Group; 6:6149.

[4] Solhan M.B., Trull T.J., Jahng S., Wood P.K., 2009. Clinical Assessment of Affective Instability: Compating EMA indices, questionnaire reports, and retrospective recall. Psychol Assess.; 21(3):425 36. 